\title{
Anhedonia and the Brain Reward Circuitry in Depression
}

\author{
Mitra Heshmati ${ }^{1} \cdot$ Scott J. Russo ${ }^{1}$
}

Published online: 12 July 2015

(C) Springer International Publishing AG 2015

\begin{abstract}
Anhedonia, or the loss of pleasure in previously rewarding stimuli, is a core symptom of major depressive disorder that may reflect an underlying dysregulation in reward processing. The mesolimbic dopamine circuit, also known as the brain's reward circuit, is integral to processing the rewarding salience of stimuli to guide actions. Manifestation of anhedonia and associated depression symptoms, like feelings of sadness, changes in appetite, and psychomotor effects, may reflect changes in the brain reward circuitry as a common underlying disease process. This review will synthesize the recent literature from human and rodent studies providing a circuit-level framework for understanding anhedonia in depression, with emphasis on the nucleus accumbens.
\end{abstract}

Keywords Nucleus accumbens $\cdot$ Depression $\cdot$ Reward circuitry $\cdot$ Medium spiny neuron $\cdot$ Dopamine $\cdot$ Optogenetics . Anhedonia $\cdot$ Chronic social defeat stress

\section{Introduction}

One in six Americans suffer from major depressive disorder (MDD) [1,2], yet it is estimated that one-third of MDD patients treated with current therapies are treatment-resistant [3]. There

This article is part of the Topical Collection on Mood and Anxiety Disorders

Scott J. Russo

scott.russo@mssm.edu

1 Fishberg Department of Neuroscience, Friedman Brain Institute, Graduate School of Biomedical Sciences, Icahn School of Medicine at Mount Sinai, 1425 Madison Avenue, Icahn 10-71, Box 1065, New York, NY 10029, USA is an urgent need for more effective therapeutic approaches, and a better understanding of the pathophysiology of depression will present novel treatment strategies. Depressed patients must display one of two core symptoms of depression: anhedonia or feelings of sadness. Studies have shown that anhedonia and reduced reward learning [4] predict a poorer prognosis [5], with a higher prevalence of treatment failure among anhedonic patients [6]. The number of depressed patients with anhedonia suggests that aberrant reward processing, perhaps due to changes in reward circuitry, is a core mechanism underlying depression pathophysiology [7]. Recent neuroimaging studies in depressed patients show reduced activation of the reward circuitry in depression, specifically the nucleus accumbens (NAc) and anterior cingulate cortex (ACC) [8]. Several studies have shown that deep brain stimulation of either the NAc or ACC ameliorates anhedonia in treatment-refractory depression $[9,10]$. A greater understanding of the cellular mechanisms within brain reward circuitry is needed to provide insights into the anhedonic state and reveal new therapeutic targets.

A major challenge facing basic science research in the area of mood disorders is the difficulty in modeling human psychiatric symptoms like guilt, suicidality, and sadness in rodents $[11,12]$. In addition, unlike other psychiatric illnesses, recent genome-wide association studies have yielded no clear genetic linkages to depression, preventing the field from developing genetic models of the disease. It is long known that chronic stress is a predisposing risk factor for depression [13] and thus basic research has focused on developing models of chronic stress to recapitulate aspects of the disease in rodents. These rodent depression models are yielding fundamental insights into the basic mechanisms that influence depression vulnerability or resilience $[14,15]$, as well as enabling a dissection of the reward circuitry using optogenetic techniques [16, 17]. Coupled to neuroimaging and deep brain stimulation studies in humans $[18,19]$, these data are providing novel insight into 
the brain reward circuitry in depression. Here, we will summarize recent investigations of the brain reward circuit using animal models of depression and anhedonia, with a focus on the nucleus accumbens circuitry.

\section{Animal Models of Depression}

In order to study depression and anhedonia in rodents, most models examine chronic stress effects on natural rewards like social interaction and sucrose consumption. The chronic social defeat stress model repeatedly exposes young adult male mice to a series of antagonistic encounters with an older, more aggressive male mouse, resulting in a range of depression-like phenotypes [14, 20]. All of the mice that undergo social defeat stress display anxiety-like behavior in exploratory-based tasks, but they are differentiated by deficits in social interaction behavior and sucrose preference, an established measure of anhedonia in mice [21, 22]. In addition to sucrose preference, intracranial self-stimulation (ICSS) is used to measure anhedonia. Elevation in ICSS threshold indicates reduced reward circuit function, while increased ICSS suggests increased reward seeking. Interestingly, chronic social defeat stress causes elevations in ICSS threshold [23, 24].

Approximately two-thirds of experimental mice that undergo social defeat stress display social avoidance and reduced sucrose preference and are termed susceptible. One-third of mice do not exhibit anhedonia, as measured by reduced sucrose consumption, or display deficits in social interaction and are termed resilient [25]. This finding is analogous to diverse human populations, whereby only a subset of individuals subjected to daily life stress develop depression. As a measure of predictive validity, only chronic 30-day treatment with imipramine is sufficient to reverse the social avoidance characteristic of susceptible mice. While most antidepressant drug development efforts have focused on reversing pathophysiological changes observed in susceptible individuals, recent studies have also shown that resiliency to stress is not merely the lack of susceptibility but requires the engagement of active mechanisms [15, 26-28]. Thus, the chronic social defeat stress model enables the analysis of mechanisms that confer either susceptibility or resiliency to stress. A disadvantage of the model is its restriction to males, since female mice do not commonly display similar antagonistic interactions (an important exception is California mice [29]). Other models, like chronic mild stress or chronic unpredictable stress, may be better suited for studying sex differences. In chronic mild stress, animals undergo a month of daily, variable stressors ranging from wet bedding to restraint to tail suspension or foot shock. Protocols vary, but the accumulation of mild unpredictable stressors over a long period of time results in anhedonia, anxiety, reduced social interaction, and behavioral despair
[30]. Since depression is more prevalent in women, it is necessary in the future to consider additional models that enable the study of sex differences in stress susceptibility [31]. The majority of studies that will be discussed in this review use either the chronic social defeat stress or chronic mild stress paradigm in mice to model facets of anhedonia. We refer the reader to reviews that analyze the utility of animal models in more detail [11, 32].

\section{Brain Reward Circuit}

The mesolimbic reward circuit consists primarily of the prefrontal cortex (PFC), nucleus accumbens (NAc), ventral tegmental area (VTA), amygdala (AMY), and hippocampus (HIP) [33, 34] (See Fig. 1). Additional brain regions like the lateral hypothalamus $(\mathrm{LH})[35,36]$, lateral habenula (LHb) [37], and dorsal striatum [38] also play important roles in the reward circuit but will not be discussed here. This review will focus on the glutamatergic and dopaminergic circuitry that directly impinges on the NAc, due to the integral role of the NAc in the reward circuit.

The NAc is a key brain reward region in the ventral striatum that integrates different excitatory and inhibitory inputs to signal the salience of rewarding stimuli [39]. The NAc receives heterogeneous dopaminergic and GABAergic projections from the ventral tegmental area $[40,41]$ as well as glutamatergic afferents from the thalamus [42•], PFC [43, 44], HIP [45, 46], and AMY [47]. In turn, the NAc sends GABAergic projections to the VTA and ventral pallidum. The primary output of the NAc is then relayed through the mediodorsal nucleus of the thalamus to cortex to modulate responses to reward-related cues [48]. Recently, a direct cortical projection from cholinergic cells in the striatum was identified [49], and it is possible that direct cortical projections from NAc interneurons also exist. In these ways, the NAc is a complex, integral hub in the cortico-limbic circuit.

\section{PFC to NAc Circuit}

The popular theory of hypofrontality in depression is based upon observations of decreased cerebral blood flow and decreased activation of prefrontal cortical regions in studies of patients with major depressive disorder [50-53]. Deep brain stimulation of the human subgenual anterior cingulate cortex was also shown to improve treatment-refractory depression [54]. Comparatively, chronic stress paradigms in rodents result in atrophy and decreased spine synapses in the medial prefrontal cortex [55-57]. In a recent study in mice, deep brain stimulation of the ventromedial PFC after chronic social defeat stress (CSDS) reversed social avoidance behavior and stress effects on serotonergic neurons [58]. From these studies, it was not clear which cell type and output region of the PFC was responsible for these antidepressant effects; however, 


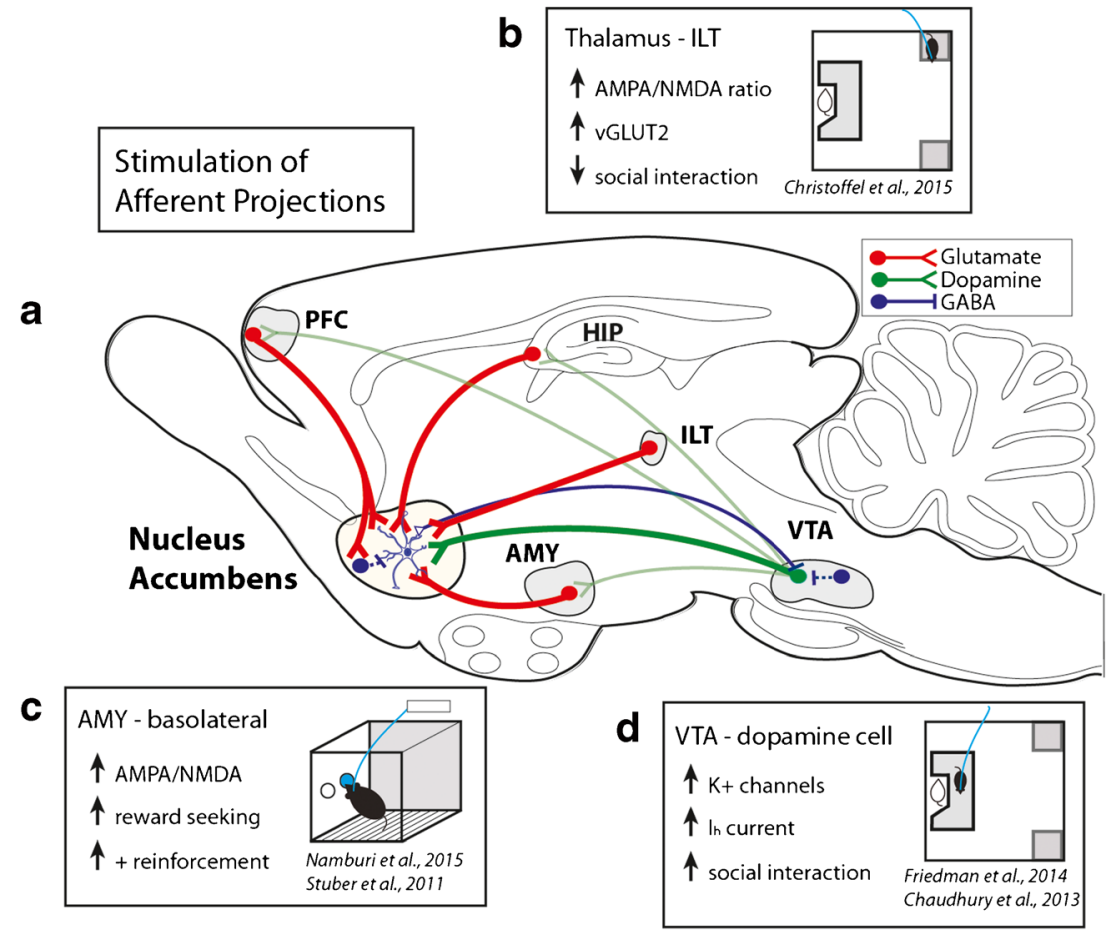

Fig. 1 a This simplified schematic depicts the major glutamatergic afferents (red) to nucleus accumbens ( $N A C$ ) medium spiny neurons. The VTA sends dopaminergic projections to NAc (green) as well as PFC and HIP (light green). Interneurons and NAc medium spiny neurons provide GABAergic input (blue). The lateral hypothalamus, lateral habenula, dorsal striatum, and ventral pallidum have been omitted for clarity, though they also play important roles in the reward circuit. Ventral tegmental area $(V T A)$, amygdala $(A M Y)$, prefrontal cortex $(P F C)$, hippocampus $(H I P)$, and intralaminar thalamus $(I L T)$. b Optogenetic stimulation of glutamatergic thalamostriatal synapses increased social avoidance behavior in a recent study by Christoffel et al. [42•]. After chronic social defeat stress, susceptible mice displayed baseline increases in the strength of thalamic synapses in the ventral striatum, as measured by increased vesicular glutamate transporter- 2 protein and increased AMPA to NMDA receptor ratio on medium spiny neurons with optogenetic stimulation of thalamic terminals. $\mathbf{c}$ The basolateral amygdala $(B L A)$ projection to NAc signaled positive reward reinforcement, and stimulation of this projection promoted intracranial self-stimulation (ICSS) in a recent study by Namburi et al. [70•]. The study expanded upon earlier work showing the rewarding effects of BLA to NAc terminal stimulation [47]. After sucrose reward conditioning, BLA neurons that project to the NAc displayed increased synaptic strength as measured by increased AMPA to NMDA ratio. In an ICSS task, animals will nosepoke for optogenetic stimulation of BLA neurons that project to the NAc. d VTA dopamine neuron firing and $\mathrm{I}_{\mathrm{h}}$ current was previously shown to increase in susceptible animals after chronic social defeat stress [14]. A recent study demonstrated that resilient mice have even larger $I_{h}$ currents along with increased potassium $(\mathrm{K}+)$ channel currents [77••]. Enhancing this resiliency mechanism by stimulating dopamine cells or by increasing potassium channel currents promoted increased social interaction earlier studies showed a reduction in immediate early gene expression both in the prefrontal cortex of depressed patients and susceptible mice after CSDS [59] that appeared to be glutamatergic. High-frequency optogenetic stimulation of prefrontal cortical glutamatergic afferents increased c-Fos expression in the NAc and reversed social avoidance behavior after CSDS [59]. Recent studies elaborated on these findings, showing that high-frequency optogenetic stimulation of PFC inputs to NAc reverses social avoidance in a mechanism involving cholecystokinin blockade [42•, 60]. Stimulation of PFC inputs to the amygdala had an anxiogenic effect but did not produce the same reversal in social avoidance behavior [60].

As mentioned above, these studies were performed using high-frequency, $100 \mathrm{~Hz}$, stimulation of PFC to NAc synapses, and it is unclear using these parameters whether the stimulation is selective to PFC terminals or if antidromic activity may have affected the behavioral results. Lower frequency stimulation of PFC to NAc terminals with channelrhodopsin or silencing with halorhodopsin does not affect social avoidance [42•]. While the specific role of PFC to NAc projections in susceptibility to stress is still unclear, we recently found that thalamic projections to ventral striatum in susceptible mice displayed increased excitatory strength as measured by increased AMPA/NMDA ratio and increased vesicular glutamate transporter-2 after stress. Activation of the thalamostriatal glutamatergic projections to NAc was found to be necessary and sufficient for social avoidance behavior following CSDS [42•].

\section{HIP to NAc Circuit}

The hippocampus (HIP) receives and sends inputs to a number of different brain regions in the reward circuit, including the 
VTA, AMY, and NAc. Due to this reciprocal connectivity, it also plays important and complex roles in the processing of reward valence. Emotionally salient events are suggested to form stronger memories, and this relies in part on interactions between the AMY and HIP [61, 62]. Patients with MDD show decreased performance in several tasks of memory and cognition $[63,64]$. Dendritic spine atrophy in CA1 and CA3 pyramidal cells of the hippocampus as well as decreased neurogenesis have been well documented in rodent studies of chronic stress [65]. In contrast, increased adult neurogenesis in the hippocampus of a transgenic mouse model resulted in reduced anxiety and depression-like behaviors [66]. Further, a recent study examined ventral hippocampal afferents to the nucleus accumbens, finding that optogenetic stimulation of these terminals in the NAc promoted susceptibility to chronic social defeat stress [46]. Resilient mice showed reduced ventral hippocampal activity following stress, and optogenetic induction of long-term depression at ventral hippocampal to NAc synapses promoted resiliency [46].

\section{AMY to NAc Circuit}

The amygdala (AMY) is heavily implicated in depression and stress behaviors, due to its role in modulating fear-mediated responses. Functional imaging studies suggest that the AMY is overactive in depressed humans [52,53], and rodent stress studies show increased dendritic spine density in the basolateral amygdala (BLA) [67]. Interestingly, circuitspecific studies paint a more complicated role for the AMY in reward and anxiety. Increased firing of the BLA precedes increased cue-evoked firing in the NAc, and the BLA to NAc projection contributes to reward seeking [68]. In mouse optogenetic studies, stimulation of BLA terminals in the NAc promoted increased reward seeking [47]. On the other hand, activation of BLA to central nucleus projections decreased anxiety while inhibition of BLA terminals in the central nucleus of the amygdala (CeA) was anxiogenic [69]. A recent study further investigated distinct projections from the BLA, showing that projections to the NAc are positively reinforcing while projections to the centromedial nucleus are negatively reinforcing in a fear-conditioning task compared to reward conditioning [70•]. Neurons in the BLA that projected to NAc displayed increased synaptic strength after conditioning of a tone to sucrose delivery and optogenetic stimulation of these cells increased intracranial self-stimulation, providing more evidence for a role of the AMY in reward-seeking behavior [70•].

\section{VTA to NAc Circuit}

VTA dopamine neurons are widely studied for their role in encoding reward and reinforcement learning [71]. Dopamine neurons shift from a baseline tonic firing to phasic firing in response to unexpected rewards and will encode reward prediction errors $[72,73]$. So, the idea that aberrant dopamine signaling subserves the anhedonic state has long been entertained. Initial studies using the chronic social defeat stress model in mice reported increased brain-derived neurotrophic factor (BDNF) in the VTA of susceptible mice, and selective knockout of BDNF in the VTA prevented social avoidance behavior [74]. These early studies did not distinguish between subtypes of dopamine neurons. Using optogenetic methods in mice that enable millisecond control of neuronal firing, projection-specific circuits from VTA dopamine neurons are now being investigated for having distinct depression-modulatory roles $[75,76 \bullet \bullet, 77 \bullet \bullet, 78,79 \bullet]$.

Following chronic social defeat stress, mice that are susceptible to stress display increased VTA dopamine neuron firing and large, inward hyperpolarization $\left(\mathrm{I}_{\mathrm{h}}\right)$ currents [14]. Enhanced phasic firing of dopamine neurons, using optogenetic stimulation specifically of VTA neurons that project to the NAc, induced stress susceptibility [76••]. This effect was not seen when stimulating VTA dopamine neurons that project to PFC, suggesting a specific role of dopaminergic projections to the NAc in promoting susceptibility to stress. In a recent study, resilient mice were shown to engage an active, homeostatic mechanism that involves upregulation of potassium channels and larger $\mathrm{I}_{\mathrm{h}}$ currents than seen in susceptible mice in VTA dopamine neurons following stress [77••]. Optogenetic stimulation of the VTA to mimic these $I_{h}$ currents in susceptible mice promoted stress resilience [77••]. Overall, these studies suggest that both stress resilience and susceptibility involve active mechanisms, and the enhancement of certain resilience mechanisms could promote antidepressant effects.

In contrast, studies using the chronic mild stress model in mice observed opposite effects of VTA to NAc phasic stimulation on social interaction behavior [79•]. Increased phasic stimulation of VTA dopamine to NAc projections increased sucrose preference and mobility in the tail suspension test following chronic mild stress, suggesting a pro-resiliency effect counter to the studies in chronic social defeat stress [76••, 79•]. The difference in behavioral paradigms could account for this dichotomy. Chronic mild stress was shown to reduce VTA dopamine neuron firing, while social defeat stress increased baseline dopamine neuron firing in susceptible mice [14, 79•]. It will be important in the future to reconcile the discrepancies in these studies in order to more fully understand the role of VTA to NAc projections in depression and anhedonia.

The discovery of a GABAergic projection from the VTA to NAc provided an additional layer of complexity in deciphering VTA to NAc projections [80]. Indiscriminate optogenetic stimulation of VTA to NAc projections may indeed modulate these GABAergic projections. The previously highlighted studies were performed in mice that expressed $\mathrm{Cre}$ 
recombinase in tyrosine hydroxylase-positive cells, enabling conditional activation of dopaminergic cells. However, the specificity of channelrhodopsin expression in this Th-Cre transgenic mouse line was recently disputed [81, 82•]. Studies that specifically activated VTA gamma aminobutyric acid (GABA) neurons showed a decrease in dopamine release in the NAc, but no effect on reward behavior as measured by sucrose consumption [83].

GABAergic projections from VTA directly innervate cholinergic interneurons in the nucleus accumbens [41]. In addition, phasic dopamine release controls cholinergic tone via D2 and D1 receptor activation on cholinergic interneurons [84]. Since cholinergic interneurons have been shown to elicit inhibitory currents in striatal medium spiny neurons $[85,86]$, the net result of VTA GABA projections may involve disinhibition of medium spiny neurons. However, cholinergic cells also synapse on local GABAergic interneurons, for example eliciting responses in neuropeptide Y-positive interneurons, so there may be additional synaptic modulation by GABAergic interneurons changing the net functional output of these projections [85]. Clearly, more work is needed, particularly in the ventral striatum, to better understand the differential effects of dopaminergic and GABAergic VTA inputs on medium spiny neuron outputs and the behavioral effects in depression models.

\section{NAc Microcircuit}

In addition to the many afferent inputs, the nucleus accumbens itself contains an intricate inhibitory microcircuit that adds an additional layer of complexity to the processing of rewardrelated stimuli (Fig. 1). Medium spiny neurons (MSNs) are the principal GABAergic cells in the NAc, comprising approximately $95-97 \%$ of the total cell population in rodents and $85 \%$ in primates [87]. Although they synthesize and release gamma aminobutyric acid (GABA) as a neurotransmitter, MSNs are spiny, projection neurons. They are also intricately connected to each other, creating a network of local inhibitory collaterals $[88,89]$ in addition to inhibitory regulation by GABAergic and cholinergic interneurons [90-93].

MSNs are distinguished by their dopamine receptor expression profiles, analogous to their counterparts in dorsal striatum [94]. Most MSNs predominantly express either the dopamine D1 receptor (Drd1) or dopamine D2 receptor (Drd2) and will be referred to as D1-MSNs and D2-MSNs, respectively. D1-MSNs directly innervate the VTA while D2-MSNs signal through the ventral pallidum to VTA, forming the direct and indirect pathways, respectively. However, there is evidence of MSN subpopulations which coexpress both Drd1 and Drd2 subtypes [95, 96]. The role of these overlapping dopamine receptor populations in anhedonia and depression is still unclear.
D1-MSNs and D2-MSNs are known to have distinct functional roles in the circuitry, so it is important to differentiate between these cell populations when studying the NAc. Selective channelrhodopsin-mediated optogenetic stimulation of D1-MSNs was shown to increase the rewarding effects of cocaine, as well as to promote resilience following social defeat stress [97, 98]. On the other hand, repeated, daily stimulation of D2-MSNs during the chronic social defeat stress paradigm promoted social avoidance. Unlike D1-MSN optogenetic stimulation, D2-MSN stimulation did not produce rapid behavioral effects [97], suggesting a complex and delayed mechanism underlying D2-MSN-mediated social avoidance behavior. Further studies are needed to understand the cellular adaptations important in mediating these delayed effects of D2 activation in stress models.

Previous studies have shown that multiple chronic stress paradigms, including gestational stress, chronic mild stress, and chronic social defeat stress, produce an increase in excitatory synaptic tone and synaptic remodeling in the NAc of susceptible mice [99-103]. In the social defeat stress model, susceptible mice had increased density of stubby, immature spines, along with increased mini excitatory postsynaptic current (mEPSC) frequency, but no change in amplitude of currents [99], suggesting that there are more functional excitatory synapses on NAc MSNs. Further studies showed that the increase in mEPSC frequency is restricted to D2-MSNs in the NAc [97]. Overall, these data suggest a model in which increased excitatory afferents to NAc D2-MSNs induces synaptic plasticity that underlies susceptibility to stress. The shift in excitatory to inhibitory tone in this circuit may reflect a failure in homeostatic mechanisms to upregulate inhibition in susceptible animals.

Although MSNs comprise the major population of cells in the NAc, subpopulations of either GABAergic or cholinergic interneurons are present as well and are known to contribute to the overall tone of the microcircuitry [33,34]. The main classes of GABAergic interneurons are classified partly based on expression of calcium-binding proteins and neuropeptides: (1) mostly overlapping population of neuropeptide $\mathrm{Y}$, nitric oxide synthase, and somatostatin-expressing cells, (2) parvalbumin, and (3) calbindin-2 or calretinin [87]. Each of these GABAergic interneuron populations also has a distinct electrophysiological profile [92, 104]. The large, cholinergic interneuron population is marked by expression of choline acetyltransferase (ChAT) and exhibits tonically active, pause-excitation firing [84]. Silencing cholinergic interneurons in the NAc was shown to promote depression-like behaviors in one study [93]. Parvalbumin-positive neurons are fast-spiking interneurons that form proximal somatic synapses, while neuropeptide $\mathrm{Y}$ and somatostatin-expressing cells are classified as persistent low-threshold spiking and often synapse on the distal dendrites [104]. Despite the sparsity of GABAergic interneurons, a dense dendritic tree 
enables one interneuron to contact hundreds of MSNs and provide powerful inhibitory control [105]. Although emerging reports are beginning to define the roles of the inhibitory interneurons in the NAc, far more work is needed in this area.

\section{Conclusion}

The brain's reward circuit is critical in parsing positive and negative stimuli, and it is increasingly appreciated that dysfunction within the circuit manifests as psychiatric symptoms such as anhedonia, or an inability to normally experience rewards in one's environment. Within the reward circuit, the NAc serves as an integral hub that receives inhibitory synaptic inputs from other MSNs in the form of distal dendritic inhibitory collateral synapses, along with inhibitory synaptic inputs from interneurons [88]. There is also additional inhibitory input from the VTA GABAergic population. However, the majority of synapses within the NAc are glutamatergic and arise from the many excitatory afferents previously discussed from PFC, AMY, and HIP [106]. As detailed above, the integration of circuit-specific afferent inputs into the NAc inhibitory network may ultimately determine reward-mediated behavioral outcomes following exposure to chronic stress. Unraveling the complexity of this integration is the next challenge in developing cell-targeted therapies for anhedonia and depression.

Acknowledgements $\mathrm{MH}$ and SJR receive grant funding from the US National Institute of Mental Health: RO1MH090264 (to SJR) and F30MH100835 (to MH).

\section{Compliance with Ethics Guidelines}

Conflict of Interest Scott Russo and Mitra Heshmati have no relevant disclosures to report.

Human and Animal Rights and Informed Consent This article does not contain any studies with human or animal subjects performed by any of the authors.

\section{References}

Papers of particular interest, published recently, have been highlighted as:

- Of Importance

•- Of major importance

1. Kessler RC, Wang PS. The descriptive epidemiology of commonly occurring mental disorders in the United States. Annu Rev Public Health. 2008;29:115-29.

2. Greenberg PE et al. The economic burden of adults with major depressive disorder in the United States (2005 and 2010). J Clin Psychiatry. 2015;76(2):155-62.
3. Rush AJ et al. Acute and longer-term outcomes in depressed outpatients requiring one or several treatment steps: a STAR*D report. Am J Psychiatry. 2006;163(11):1905-17.

4. Vrieze E et al. Reduced reward learning predicts outcome in major depressive disorder. Biol Psychiatry. 2013;73(7):639-45.

5. Vrieze E et al. Dimensions in major depressive disorder and their relevance for treatment outcome. J Affect Disord. 2014;155:3541.

6. McMakin DL et al. Anhedonia predicts poorer recovery among youth with selective serotonin reuptake inhibitor treatmentresistant depression. J Am Acad Child Adolesc Psychiatry. 2012;51(4):404-11.

7. Keedwell PA et al. The neural correlates of anhedonia in major depressive disorder. Biol Psychiatry. 2005;58(11):843-53.

8. Satterthwaite TD et al. Common and dissociable dysfunction of the reward system in bipolar and unipolar depression. Neuropsychopharmacol 2015.

9. Schlaepfer TE et al. Deep brain stimulation to reward circuitry alleviates anhedonia in refractory major depression. Neuropsychopharmacology. 2008;33(2):368-77.

10. Bewernick BH et al. Nucleus accumbens deep brain stimulation decreases ratings of depression and anxiety in treatment-resistant depression. Biol Psychiatry. 2010;67(2):110-6.

11. Nestler EJ, Hyman SE. Animal models of neuropsychiatric disorders. Nat Neurosci. 2010;13(10):1161-9.

12. McKinney Jr WT, Bunney Jr WE. Animal model of depression. I. Review of evidence: implications for research. Arch Gen Psychiatry. 1969;21(2):240-8.

13. Kessler RC. The effects of stressful life events on depression. Annu Rev Psychol. 1997;48:191-214.

14. Krishnan V et al. Molecular adaptations underlying susceptibility and resistance to social defeat in brain reward regions. Cell. 2007;131(2):391-404.

15. Russo SJ et al. Neurobiology of resilience. Nat Neurosci. 2012;15(11):1475-84.

16. Lobo MK, Nestler EJ, Covington 3rd HE. Potential utility of optogenetics in the study of depression. Biol Psychiatry. 2012;71(12):1068-74.

17. Steinberg EE et al. Illuminating circuitry relevant to psychiatric disorders with optogenetics. Curr Opin Neurobiol. 2015;30:9-16.

18. Drevets WC et al. A functional anatomical study of unipolar depression. J Neurosci. 1992;12(9):3628-41.

19. Schlaepfer TE et al. Deep brain stimulation of the human reward system for major depression - rationale, outcomes and outlook. Neuropsychopharmacology. 2014;39(6):1303-14.

20. Kudryavtseva NN, Bakshtanovskaya IV, Koryakina LA. Social model of depression in mice of C57BL/6J strain. Pharmacol Biochem Behav. 1991;38(2):315-20.

21. Papp M, Willner P, Muscat R. An animal model of anhedonia: attenuation of sucrose consumption and place preference conditioning by chronic unpredictable mild stress. Psychopharmacol (Berl). 1991;104(2):255-9.

22. Willner P. Validity, reliability and utility of the chronic mild stress model of depression: a 10-year review and evaluation. Psychopharmacol (Berl). 1997;134(4):319-29.

23. Donahue RJ et al. Effects of striatal DeltaFosB overexpression and ketamine on social defeat stress-induced anhedonia in mice. Biol Psychiatry. 2014;76(7):550-8.

24. Der-Avakian A et al. Enduring deficits in brain reward function after chronic social defeat in rats: susceptibility, resilience, and antidepressant response. Biol Psychiatry. 2014;76(7):542-9.

25. Golden SA et al. A standardized protocol for repeated social defeat stress in mice. Nat Protoc. 2011;6(8):1183-91.

26. Dias $\mathrm{C}$ et al. Beta-catenin mediates stress resilience through Dicer1/microRNA regulation. Nature. 2014;516(7529):51-5. 
27. Elliott $\mathrm{E}$ et al. Resilience to social stress coincides with functional DNA methylation of the Crf gene in adult mice. Nat Neurosci. 2010;13(11):1351-3.

28. Vialou V et al. DeltaFosB in brain reward circuits mediates resilience to stress and antidepressant responses. Nat Neurosci. 2010;13(6):745-52.

29. Trainor BC et al. Sex differences in social interaction behavior following social defeat stress in the monogamous California mouse (Peromyscus californicus). PLoS One. 2011;6(2):e17405.

30. Willner P. Chronic mild stress (CMS) revisited: consistency and behavioural-neurobiological concordance in the effects of CMS. Neuropsychobiology. 2005;52(2):90-110.

31. Seney ML, Sibille E. Sex differences in mood disorders: perspectives from humans and rodent models. Biol Sex Differ. 2014;5(1): 17.

32. Hodes GE, Russo SJ. Animal models of mood disorders. In: Charney DS, editor. Neurobiology of mental illness. 4th ed. New York: Oxford University Press; 2013. p. 411-24.

33. Russo SJ, Nestler EJ. The brain reward circuitry in mood disorders. Nat Rev Neurosci. 2013;14(9):609-25.

34. Sesack SR, Grace AA. Cortico-basal ganglia reward network: microcircuitry. Neuropsychopharmacology. 2010;35(1):27-47.

35. Nieh EH et al. Decoding neural circuits that control compulsive sucrose seeking. Cell. 2015;160(3):528-41.

36. Larson EB et al. Optogenetic stimulation of accumbens shell or shell projections to lateral hypothalamus produce differential effects on the motivation for cocaine. J Neurosci. 2015;35(8):353743.

37. Shabel SJ et al. Mood regulation. GABA/glutamate co-release controls habenula output and is modified by antidepressant treatment. Science. 2014;345(6203):1494-8.

38. Isomura $\mathrm{Y}$ et al. Reward-modulated motor information in identified striatum neurons. J Neurosci. 2013;33(25):10209-20.

39. Smith KS, Berridge KC, Aldridge JW. Disentangling pleasure from incentive salience and learning signals in brain reward circuitry. Proc Natl Acad Sci U S A. 2011;108(27):E255-64.

40. Tritsch NX, Ding JB, Sabatini BL. Dopaminergic neurons inhibit striatal output through non-canonical release of GABA. Nature. 2012;490(7419):262-6.

41. Brown MT et al. Ventral tegmental area GABA projections pause accumbal cholinergic interneurons to enhance associative learning. Nature. 2012;492(7429):452-6.

42. Christoffel DJ et al. Excitatory transmission at thalamo-striatal synapses mediates susceptibility to social stress. Nat Neurosci. 2015;18(7):962-4. This manuscript is the first to implicate an increase in excitatory drive at thalamo-striatal synapses in depression.

43. Montaron MF et al. Prefrontal cortex inputs of the nucleus accumbens-nigro-thalamic circuit. Neuroscience. 1996;71(2): 371-82.

44. Ballard IC et al. Dorsolateral prefrontal cortex drives mesolimbic dopaminergic regions to initiate motivated behavior. J Neurosci. 2011;31(28):10340-6.

45. Britt JP et al. Synaptic and behavioral profile of multiple glutamatergic inputs to the nucleus accumbens. Neuron. 2012;76(4): 790-803.

46. Bagot RC et al. Ventral hippocampal afferents to the nucleus accumbens regulate susceptibility to depression. Nat Commun. 2015;6:7062.

47. Stuber GD et al. Excitatory transmission from the amygdala to nucleus accumbens facilitates reward seeking. Nature. 2011;475(7356):377-80

48. Leung BK, Balleine BW. Ventral pallidal projections to mediodorsal thalamus and ventral tegmental area play distinct roles in outcome-specific pavlovian-instrumental transfer. J Neurosci. 2015;35(12):4953-64.
49. Saunders A et al. A direct GABAergic output from the basal ganglia to frontal cortex. Nature 2015.

50. Galynker II et al. Hypofrontality and negative symptoms in major depressive disorder. J Nucl Med. 1998;39(4):608-12.

51. Elliott $\mathrm{R}$ et al. Reduced medial prefrontal responses to social interaction images in remitted depression. Arch Gen Psychiatry. 2012;69(1):37-45.

52. Zhong $\mathrm{M}$ et al. Amygdala hyperactivation and prefrontal hypoactivation in subjects with cognitive vulnerability to depression. Biol Psychol. 2011;88(2-3):233-42.

53. Siegle GJ et al. Increased amygdala and decreased dorsolateral prefrontal BOLD responses in unipolar depression: related and independent features. Biol Psychiatry. 2007;61(2):198-209.

54. Johansen-Berg $\mathrm{H}$ et al. Anatomical connectivity of the subgenual cingulate region targeted with deep brain stimulation for treatment-resistant depression. Cereb Cortex. 2008;18(6):1374 83.

55. Shansky RM et al. Stress-induced dendritic remodeling in the prefrontal cortex is circuit specific. Cereb Cortex. 2009;19(10): 2479-84.

56. Radley JJ et al. Repeated stress induces dendritic spine loss in the rat medial prefrontal cortex. Cereb Cortex. 2006;16(3):313-20.

57. Radley JJ et al. Repeated stress alters dendritic spine morphology in the rat medial prefrontal cortex. J Comp Neurol. 2008;507(1): 1141-50.

58. Veerakumar A et al. Antidepressant-like effects of cortical deep brain stimulation coincide with pro-neuroplastic adaptations of serotonin systems. Biol Psychiatry. 2014;76(3):203-12.

59. Covington $3 \mathrm{rd}$ HE et al. Antidepressant effect of optogenetic stimulation of the medial prefrontal cortex. J Neurosci. 2010;30(48): 16082-90.

60. Vialou V et al. Prefrontal cortical circuit for depression- and anxiety-related behaviors mediated by cholecystokinin: role of DeltaFosB. J Neurosci. 2014;34(11):3878-87.

61. Richardson MP, Strange BA, Dolan RJ. Encoding of emotional memories depends on amygdala and hippocampus and their interactions. Nat Neurosci. 2004;7(3):278-85.

62. Yonelinas AP, Ritchey M. The slow forgetting of emotional episodic memories: an emotional binding account. Trends Cogn Sci. 2015;19(5):259-67.

63. Fairhall SL et al. Memory related dysregulation of hippocampal function in major depressive disorder. Biol Psychol. 2010;85(3): 499-503.

64. Soderlund $\mathrm{H}$ et al. Autobiographical episodic memory in major depressive disorder. J Abnorm Psychol. 2014;123(1):51-60.

65. McEwen BS. Stress, sex, and neural adaptation to a changing environment: mechanisms of neuronal remodeling. Ann N Y Acad Sci. 2010;1204(Suppl):E38-59.

66. Hill AS, Sahay A, Hen R. Increasing adult hippocampal neurogenesis is sufficient to reduce anxiety and depression-like behaviors. Neuropsychopharmacol 2015.

67. Mitra $\mathrm{R}$ et al. Stress duration modulates the spatiotemporal patterns of spine formation in the basolateral amygdala. Proc Natl Acad Sci U S A. 2005;102(26):9371-6.

68. Ambroggi $\mathrm{F}$ et al. Basolateral amygdala neurons facilitate rewardseeking behavior by exciting nucleus accumbens neurons. Neuron. 2008;59(4):648-61.

69. Tye KM et al. Amygdala circuitry mediating reversible and bidirectional control of anxiety. Nature. 2011;471(7338):358-62.

70. Namburi $\mathrm{P}$ et al. A circuit mechanism for differentiating positive and negative associations. Nature. 2015;520(7549):675-8. This study along with Ref 47 dissects amygdala projections to the nucleus accumbens using optogenetics. It supports a role for BLA to NAc projections in positive reinforcement and distinguishes these afferents from BLA projections to the centromedial amygdala. 
71. Wise RA. Dopamine, learning and motivation. Nat Rev Neurosci. 2004;5(6):483-94.

72. Schultz W. The phasic reward signal of primate dopamine neurons. Adv Pharmacol. 1998;42:686-90.

73. Schultz W. Predictive reward signal of dopamine neurons. J Neurophysiol. 1998;80(1):1-27.

74. Berton $\mathrm{O}$ et al. Essential role of BDNF in the mesolimbic dopamine pathway in social defeat stress. Science. 2006;311(5762): 864-8.

75. Walsh JJ, Han MH. The heterogeneity of ventral tegmental area neurons: projection functions in a mood-related context. Neuroscience. 2014;282C:101-8.

$76 . \bullet$ Chaudhury D et al. Rapid regulation of depression-related behaviours by control of midbrain dopamine neurons. Nature. 2013;493(7433):532-6. This study uses circuit-specific optogenetics to confirm that increased phasic firing of VTA dopamine neurons projecting to the NAc has a role in in mediating stress susceptibility.

77.• Friedman AK et al. Enhancing depression mechanisms in midbrain dopamine neurons achieves homeostatic resilience. Science. 2014;344(6181):313-9. This study defines an active mechanism of resiliency in VTA dopamine neurons that project to the NAc and enhancing this mechanism using optogenetic stimulation promotes increased social interaction.

78. Walsh JJ et al. Stress and CRF gate neural activation of BDNF in the mesolimbic reward pathway. Nat Neurosci. 2014;17(1):27-9.

79. Tye KM et al. Dopamine neurons modulate neural encoding and expression of depression-related behaviour. Nature. 2013;493(7433):537-41. Along with reference 82, this paper highlights the complexity of reward circuit function in stress disorders. Contrary to the increase in dopamine neuron firing observed after social defeat, these authors find that chronic mild stress decreases dopamine firing to promote depressionlike behaviour.

80. Van Bockstaele EJ, Pickel VM. GABA-containing neurons in the ventral tegmental area project to the nucleus accumbens in rat brain. Brain Res. 1995;682(1-2):215-21.

81. Lammel S et al. Diversity of transgenic mouse models for selective targeting of midbrain dopamine neurons. Neuron. 2015;85(2): 429-38.

82. Stuber GD, Stamatakis AM, Kantak PA. Considerations when using cre-driver rodent lines for studying ventral tegmental area circuitry. Neuron. 2015;85(2):439-45. Along with Reference 81, this study identifies inherent problems with traditional Cre drivers lines when attempting to target specific cell types. They find ectopic expression of Cre in a small subset of non dopaminergic neurons in the VTA.

83. van Zessen R et al. Activation of VTA GABA neurons disrupts reward consumption. Neuron. 2012;73(6):1184-94.

84. Wieland $\mathrm{S}$ et al. Phasic dopaminergic activity exerts fast control of cholinergic interneuron firing via sequential NMDA, D2, and D1 receptor activation. J Neurosci. 2014;34(35):11549-59.

85. English DF et al. GABAergic circuits mediate the reinforcementrelated signals of striatal cholinergic interneurons. Nat Neurosci. 2012;15(1):123-30.

86. Nelson AB et al. Striatal cholinergic interneurons Drive GABA release from dopamine terminals. Neuron. 2014;82(1):63-70.

87. Kawaguchi Y et al. Striatal interneurones: chemical, physiological and morphological characterization. Trends Neurosci. 1995;18(12):527-35.
88. Taverna S, Ilijic E, Surmeier DJ. Recurrent collateral connections of striatal medium spiny neurons are disrupted in models of Parkinson's disease. J Neurosci. 2008;28(21):5504-12.

89. Lalchandani RR et al. Dopamine D2 receptors regulate collateral inhibition between striatal medium spiny neurons. J Neurosci. 2013;33(35):14075-86.

90. Koos T, Tepper JM, Wilson CJ. Comparison of IPSCs evoked by spiny and fast-spiking neurons in the neostriatum. J Neurosci. 2004;24(36):7916-22.

91. Kohnomi S, Koshikawa N, Kobayashi M. D(2)-like dopamine receptors differentially regulate unitary IPSCs depending on presynaptic GABAergic neuron subtypes in rat nucleus accumbens shell. J Neurophysiol. 2012;107(2):692-703.

92. Gittis $\mathrm{AH}$ et al. Distinct roles of GABAergic interneurons in the regulation of striatal output pathways. J Neurosci. 2010;30(6): 2223-34.

93. Warner-Schmidt JL et al. Cholinergic interneurons in the nucleus accumbens regulate depression-like behavior. Proc Natl Acad Sci U S A. 2012;109(28):11360-5.

94. Gong $\mathrm{S}$ et al. A gene expression atlas of the central nervous system based on bacterial artificial chromosomes. Nature. 2003;425(6961):917-25.

95. Wong AC et al. D1- and D2-like dopamine receptors are colocalized on the presynaptic varicosities of striatal and nucleus accumbens neurons in vitro. Neuroscience. 1999;89(1):221-33.

96. Surmeier DJ, Yan Z, Song WJ. Coordinated expression of dopamine receptors in neostriatal medium spiny neurons. Adv Pharmacol. 1998;42:1020-3.

97. Francis TC et al. Nucleus accumbens medium spiny neuron subtypes mediate depression-related outcomes to social defeat stress. Biol Psychiatry. 2015;77(3):212-22.

98. Lobo MK et al. DeltaFosB induction in striatal medium spiny neuron subtypes in response to chronic pharmacological, emotional, and optogenetic stimuli. J Neurosci. 2013;33(47):18381-95.

99. Christoffel DJ et al. IkappaB kinase regulates social defeat stressinduced synaptic and behavioral plasticity. J Neurosci. 2011;31(1):314-21.

100. Golden SA et al. Epigenetic regulation of RAC1 induces synaptic remodeling in stress disorders and depression. Nat Med. 2013;19(3):337-44.

101. Haim A et al. The effects of gestational stress and SSRI antidepressant treatment on structural plasticity in the postpartum brain - a translational model for postpartum depression. Horm Behav 2015.

102. Warren BL et al. Altered gene expression and spine density in nucleus accumbens of adolescent and adult male mice exposed to emotional and physical stress. Dev Neurosci. 2014;36(3-4): 250-60.

103. Bessa JM et al. Stress-induced anhedonia is associated with hypertrophy of medium spiny neurons of the nucleus accumbens. Transl Psychiatry. 2013;3:e266.

104. Tepper JM et al. Heterogeneity and diversity of striatal GABAergic interneurons. Front Neuroanat. 2010;4:150.

105. Tepper JM, Wilson CJ, Koos T. Feedforward and feedback inhibition in neostriatal GABAergic spiny neurons. Brain Res Rev. 2008;58(2):272-81.

106. Shepherd GM. The synaptic organization of the brain. 5th ed. Oxford: Oxford University Press; 2004. xiv, 719 p. 